\title{
A Degradação Ambiental e seus impactos à saude humana
}

\section{Environmental Degradation and its impacts on human health \\ La degradación ambiental y sus impactos en la salud humana}

Pedro Hudson Rodrigues Teixeira ORCID: https://orcid.org/0000-0001-5909-7642 Universidade Regional do Cariri, Brasil E-mail: pedrohudson@yahoo.com.br

João Paulo Camilo de Oliveira

ORCID: https://orcid.org/0000-0003-0286-1149 Universidade Regional do Cariri, Brasi

E-mail: camilodeoliveirajoaopaulo35@gmail.com

Ednalva da Silva Santos

ORCID: https://orcid.org/0000-0002-5511-617X Universidade Regional do Cariri, Brasil E-mail: santosednalva984@gmail.com

Gabriel Messias da Silva Nascimento

ORCID: https://orcid.org/0000-0002-6487-2890 Universidade Regional do Cariri, Brasil E-mail: gabrielmessias0397@gmail.com

Leonardo Alves de Lima

ORCID: https://orcid.org/0000-0003-1295-6088 Universidade Regional do Cariri, Brasil

E-mail: leooliimaa1@gmail.com

Clêidio da Paz Cabral

ORCID: https://orcid.org/0000-0003-1825-4925 Secretaria de Educação e Esportes de Pernambuco, Brasil

E-mail: cabralbahia@hotmail.com

Cícero Jorge Verçosa

ORCID: https://orcid.org/0000-0002-3284-6719 Secretaria de Educação e Esportes de Pernambuco, Brasil

E-mail: cjvercosa@hotmail.com

Georgia Fernanda Oliveira

ORCID: https://orcid.org/0000-0002-9298-0987 Secretaria de Educação e Esportes de Pernambuco, Brasil E-mail: georgia.fernanda@gmail.com

Rosileide de Souza Costa

ORCID: https://orcid.org/0000-0002-4015-3613 Secretaria de Educação e Esportes de Pernambuco, Brasil

E-mail: rosesud@yahoo.com.br

Elizângela Maria Ferreira Ricarte

ORCID: https://orcid.org/0000-0002-2010-9981

Universidade Regional do Cariri, Brasil

E-mail: elizsalvatore10@gmail.com

Joice Layanne Guimarães Rodrigues

ORCID: https://orcid.org/0000-0002-0683-5602

Universidade Regional do Cariri, Brasil E-mail: joicelayanne17@gmail.com

André Soares Martins

ORCID: https://orcid.org/0000-0002-9463-0964

Universidade Estadual da Paraíba, Brasil

E-mail: asmandre92@gmail.com

Gledson Ferreira Macedo

ORCID: https://orcid.org/0000-0002-2609-6894 Universidade Federal do Cariri, Brasil

E-mail: gled_fer@yahoo.com.br 
Luciano Temoteo dos Santos

ORCID: https://orcid.org/0000-0002-9215-6832

Universidade Federal do Cariri, Brasil

E-mail: luciano.temoteosantos@gmail.com

Luiz Neldecílio Alves Vitor

ORCID: https://orcid.org/0000-0003-4085-4214 Secretaria de Educação e Esportes de Pernambuco, Brasil

E-mail: professorluizneldecilio@gmail.com

Jailson Renato de Lima Silva

ORCID: https://orcid.org/0000-0003-1292-8060 Universidade Regional do Cariri, Brasil E-mail: jailsonslrj@outlook.com

Wianderson Souza de Alencar

ORCID: https://orcid.org/0000-0001-9665-3476 Universidade Regional do Cariri, Brasi

E-mail: wiandersonsa@gmail.com

Nathália de Sousa Fernandes

ORCID: https://orcid.org/0000-0003-1079-5460 Universidade Regional do Cariri, Brasil

E-mail: natymusyc@gmail.com

Gabriel Venancio Cruz

ORCID: https://orcid.org/0000-0002-0006-5213 Universidade Regional do Cariri, Brasil

E-mail: gabrielvenancio02@hotmail.com

Crispiniano Macedo Pereira

ORCID: https://orcid.org/0000-0003-4334-7605 Universidade Regional do Cariri, Brasil

E-mail: crispinianop@gmail.com

Carlito Alves do Nascimento

ORCID: https://orcid.org/0000-0002-5010-9384 Universidade Regional do Cariri, Brasil

E-mail: carlitoalves624@gmail.com

Ramira Araújo Rodrigues Silva

ORCID: https://orcid.org/0000-0002-8284-7023 Universidade Regional do Cariri, Brasil

E-mail: ramiraaraujo70@gmail.com

Rafael de Moura Cardoso

ORCID: https://orcid.org/0000-0002-8240-9998

Instituto Federal de Ciência e Tecnologia, Brasi

E-mail: cardoso.eng2013@gmail.com

Janaina Freire Clementino Mendes

ORCID: https://orcid.org/0000-0003-2453-4325

Secretaria de Educação e Esportes de Pernambuco, Brasil E-mail: janainamendes86@gmail.com

Rafael Henrique Luciano dos Santos

ORCID: https://orcid.org/0000-0001-6968-6803 Secretaria de Educação e Esportes de Pernambuco, Brasil E-mail: rafaelhenrique.bio@gmail.com

Maria Eduarda Fernandes da Silva

ORCID: https://orcid.org/0000-0001-8931-3756 Universidade Regional do Cariri, Brasil

E-mail: eduarda.fernandes16@gmail.com

Paulo Henrique de Souza

ORCID: https://orcid.org/0000-0001-9814-9433 Secretaria de Meio Ambiente de Santana do Cariri, Brasil E-mail: henrique.igreja@outlook.com

Dárcio Luiz de Sousa Júnior

ORCID: https://orcid.org/0000-0002-1539-9267 Universidade Federal do Cariri, Brasil

E-mail: darciolsjr@gmail.com

Francisco Dionísio da Silva Júnior

ORCID https://orcid.org/0000-0003-1706-7810 Universidade Regional do Cariri, Brasil

E-mail: francisco.dionisio@urca.br

Cicero Emerson Serra Inácio

ORCID: https://orcid.org/0000-0002-8274-7399

Universidade Regional do Cariri, Brasil

E-mail: emerson1012@gmail.com

Francisca Palloma Matias Vila Nova

ORCID: https://orcid.org/0000-0002-6300-8865 Universidade Regional do Cariri, Brasil E-mail: palomajaninie@hotmail.com 


\title{
Resumo
}

A degradação ambienta é um dos principais problemas da atualidade, discutido constantemente pelas Organizações Mundiais de Proteção Ambiental. A erosão de algumas áreas sustentáveis, a extinção de espécies vegetais e animais causam mudanças nos fenômenos climáticos da região além da contribuição no aquecimento global. Objetivou-se com a realização desse estudo, avaliar e apresentar um diagnóstico de degradação ambiental de uma área às margens da CE292 em Crato - CE, ocasionado pela ação do homem e suas principais implicações socioambientais. De início foi realizada a visita a campo. Em seguida foi pautado informações sobre a área, como: localização, e os aspectos naturais e os principais impactos antrópicos que deveriam ser observados no local demarcado. Os dados adquiridos foram analisados a partir da metodologia de "Check List" (listagem de controle), na modalidade descritiva, constando as ações degradantes e os impactos observados. Com o estudo realizado identificou-se impactos dos meios abiótico, biótico e antrópico. Sendo listados impactos como: desmatamento, perda de biodiversidade, proliferação de vetores que transmitem doenças, erosão, desvalorização imobiliária, poluição visual, voçoroca e invasão de plantas exóticas.

Palavras-chave: Degradação ambiental; Resíduos sólidos; Educação ambiental.

\begin{abstract}
Environmental degradation is one of the main problems today, discussed constantly by the World Environmental Protection Organizations. The erosion of some sustainable areas, the extinction of plant and animal species causes changes in the region's climatic phenomena in addition to the contribution to global warming. The objective of this study was to evaluate and present a diagnosis of environmental degradation of an area on the margins of the CE-292 in Crato - CE, caused by the action of man and its main socioenvironmental implications. In the beginning, the field visit was carried out. Then, information about the area was guided, such as: location, and the natural aspects and the main anthropic impacts that should be observed in the demarcated location. The acquired data were analyzed using the "Check List" methodology, in the descriptive modality, including the degrading actions and the observed impacts. With the study carried out, impacts of the abiotic, biotic and anthropic media were identified. Being listed impacts such as: deforestation, loss of biodiversity, proliferation of vectors that transmit diseases, erosion, property devaluation, visual pollution, gullies and invasion of exotic plants.
\end{abstract}

Keywords: Ambiental degradation; Solid waste; Environmental education.

\section{Resumen}

La degradación ambiental es uno de los principales problemas en la actualidad, discutido constantemente por las Organizaciones Mundiales de Protección Ambiental. La erosión de algunas áreas sostenibles, la extinción de especies vegetales y animales provoca cambios en los fenómenos climáticos de la región además de la contribución al calentamiento global. El objetivo de este estudio fue evaluar y presentar un diagnóstico de degradación ambiental de un área en los márgenes de la CE-292 en Crato - CE, provocada por la acción del hombre y sus principales implicaciones socioambientales. Al principio se realizó la visita de campo. Luego, se orientó información sobre el área, tales como: ubicación, y los aspectos naturales y los principales impactos antrópicos que deben observarse en el lugar demarcado. Los datos adquiridos fueron analizados utilizando la metodología "Check List", en la modalidad descriptiva, incluyendo las acciones degradantes y los impactos observados. Con el estudio realizado se identificaron impactos de los medios abióticos, bióticos y antrópicos. Se enumeran impactos como: deforestación, pérdida de biodiversidad, proliferación de vectores transmisores de enfermedades, erosión, devaluación de propiedades, contaminación visual, cárcavas e invasión de plantas exóticas.

Palabras clave: Degradación ambiental; Residuos sólidos; Educación ambiental.

\section{Introdução}

O processo de degradação ambiental é qualquer impacto ligado ao desequilíbrio da fauna e flora afetando assim o ecossistema de uma população (Silva et al, 2018). A degradação ambiental vem sendo um dos principais problemas da atualidade sendo discutido constantemente pela ONU, pois a cada dia que passa o meio ambiente vem sofrendo mais degradação, acarretando problemas como: erosão de algumas áreas sustentáveis, a extinção de espécies vegetais e animais, mudanças nos fenômenos climáticos da região além da contribuição ao aquecimento global. A educação ambiental (EA) é uma ciência voltada para a conscientização do meio em que vivemos, responsável pela socio-ambientação dos indivíduos sobre os problemas ambientais que ocorrem em seu meio. Segunda a lei n 6.938 os principais objetivos da (EA) são a preservação do meio ambiente, a melhoria e a recuperação de áreas degradadas, bem como uma melhor qualidade de vida para a população ali presente (Brito \& Valverde, 2020). 
Segundo o art. $5^{\circ}$ da lei $n^{\circ} 6.938$, a sustentabilidade ambiental busca o desenvolvimento das diretrizes da Política Nacional do Meio Ambiente, assim resulta nos fatores da necessidade de racionalização do uso dos recursos naturais. A Política Nacional de Resíduos Sólidos (PNRS) é conhecida como a lei nº 12.305/10 ela faz referência aos processos ligados a organização dos países para saber como estes lidam com os resíduos sólidos de uma determina área, buscando sempre desenvolver a conscientização da população. Os resíduos sólidos são os restos sólidos ou semissólidos das atividades humanas de uma comunidade. (Reis et al, 2018).

O principal recorte espacial da área de estudo é a degradação ambiental na rodovia 292 que liga a cidade de Crato Ceará à Exu - Pernambuco. Essa área está inserida na Chapada do Araripe, uma Unidade de Conservação (UC) criada em 1997 e fica localizada na divisa entre Ceará, Piauí e Pernambuco (Brasil, 1997). A Chapada do Araripe abriga as principais espécies de animais e plantas que estão em perigo crítico de extinção. O turismo predomina bastante nessa área e a (EA) pode ser utilizada para promover a conscientização do meio ambiente e melhorar o processo de revitalização do espaço, promovendo uma transformação nas ações do homem para com seu habitat (Correia et al, 2020).

Diante do pressuposto, o presente artigo veio desenvolver uma temática relacionada ao Curso de Pós-Graduação em Educação Ambiental da Universidade Regional do Cariri-URCA. Tendo como objetivo principal avaliar a degradação ambiental de uma área urbana da cidade de Crato - CE, às margens da CE 292, sentido Pernambuco.

\section{Desenvolvimento, Meio Ambiente e Práticas Educativas}

A educação ambiental é atravessada por vários campos de conhecimento, o que a situa como uma abordagem multirreferencial, e a complexidade ambiental reflete um tecido conceitual heterogêneo, "onde os campos de conhecimento, as noções e os conceitos podem ser originários de várias áreas do saber” (Leff, 2001; Tristão, 2002).

A problemática socioambiental, ao questionar ideologias teóricas e práticas, propõe a participação democrática da sociedade na gestão dos seus recursos atuais e potenciais, assim como no processo de tomada de decisões para a escolha de novos estilos de vida e a construção de futuros possíveis, sob a ótica da sustentabilidade ecológica e a equidade social, é notória a degradação ambiental nos arredores da Chapada do Araripe só haverá o salto de qualidade ambiental quando se criar uma identidade crescente entre o significado e dimensões das práticas, com forte ênfase na relação entre degradação ambiental e desigualdade social, reforçando a necessidade de alianças e interlocuções coletivas (Pinheiro \& Braga Branquinho, 2011).

A reflexão sobre as práticas sociais, em um contexto marcado pela degradação permanente do meio ambiente e do seu ecossistema, envolve uma necessária articulação com a produção de sentidos sobre a educação ambiental. A dimensão ambiental configura-se crescentemente como uma questão que envolve um conjunto de atores do universo educativo, potencializando o engajamento dos diversos sistemas de conhecimento, a capacitação de profissionais e a comunidade universitária numa perspectiva interdisciplinar (Tagliapietra \& Carniatto, 2019).

\section{Metodologia}

\section{Área de estudo}

A área de estudo está localizada no município de Crato - Ceará com uma população estimada de 133031 habitantes, situado no sul do estado, distando $508 \mathrm{Km}$ da capital Fortaleza. A área de estudo, a CE - 292, situa-se cerca de $3 \mathrm{~km}$ do centro do município, nas coordenadas $7^{\circ} 13^{\prime} 05^{\prime}$ 'S $39^{\circ} 25^{\prime} 59^{\prime \prime} \mathrm{W}$ (Figura 1) (IBGE, 2020). O Crato faz limite ao norte com o município de Farias Brito, ao leste com os de Caririaçu, Juazeiro do Norte e Barbalha, a oeste com os de Nova Olinda e Santana do Cariri e ao sul com os de Exu e Moreilândia no estado de Pernambuco. Faz parte de 1 dos 33 municípios que compõem a Área de Proteção Ambiental - APA Chapada do Araripe. 
Figura 1. Localização do município de Crato (Ceará), onde está localizada a área de estudo.
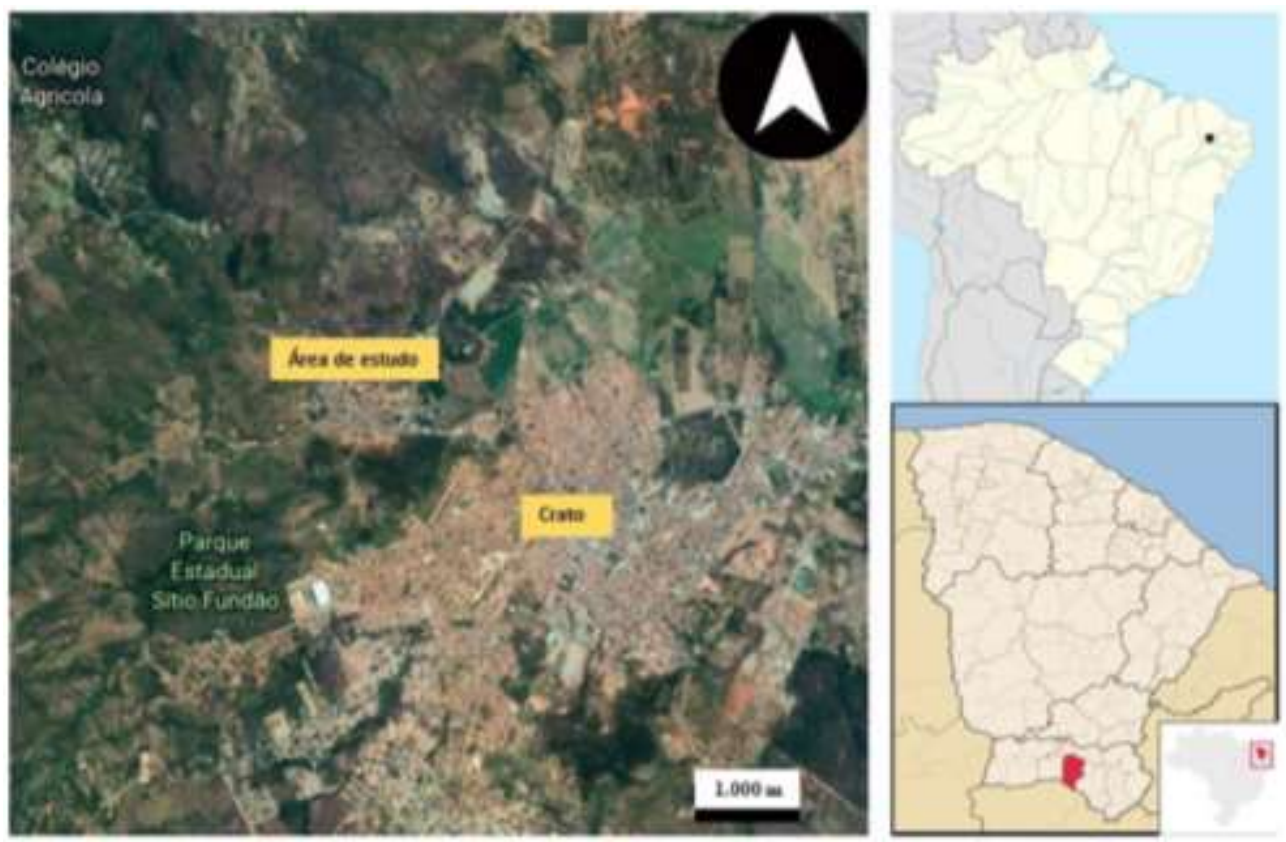

Fonte: Adaptado Google Earth (2020).

\section{Diagnóstico Prévio}

O trabalho foi realizado por alunos do curso de pós-graduação Lato Sensu em Educação Ambiental da Universidade Regional do Cariri no módulo de "Recursos naturais e Degradação Ambiental" e de início foi discutido em sala sobre o local onde seria feita a pesquisa, para se ter um melhor planejamento da visita em campo. Em seguida foi pautado informações sobre a área, como: localização, os aspectos naturais e os principais impactos antrópicos que deveriam ser observados.

\section{Diagnóstico em campo}

Foi realizada uma aula de campo na CE-292 no município de Crato no mês de Dezembro de 2018 para observar as condições em que o meio se encontrava e identificar os principais processos de degradação ambiental que ocorre no local. Com a visita foi possível observar vários impactos negativos.

\section{Caracterização e análise dos impactos}

Os dados adquiridos foram analisados a partir da metodologia de "Check List" (listagem de controle), na modalidade descritiva, constando as ações degradantes e os impactos observados. Os dados somente foram analisados quantitativamente, sendo utilizado a matriz Ponce (2011), metodologia que relaciona as ações com os fatores ambientais. Os critérios utilizados para a qualificação foram os que seguem na Tabela 1. 
Tabela 1. Critérios qualitativos para análise dos impactos.

$\begin{array}{lll}\text { CRITÉRIO PARÂMETROS } & \text { SIGNIFICADO }\end{array}$

\begin{tabular}{|c|c|c|}
\hline Ordem & $\begin{array}{l}\text { Direto: } \\
\text { Indireto: }\end{array}$ & $\begin{array}{l}\text { Quando o impacto é considerado primário. } \\
\text { Quando o impacto é uma reação secundária da ação que está sendo } \\
\text { realizada. }\end{array}$ \\
\hline & Reversível: & Quando o fator ambiental impactado, pode serrecuperado. \\
\hline \multirow[t]{2}{*}{ Plástica } & Irreversível: & Quando o fator ambiental, não é passível derecuperação. \\
\hline & Positivo: & Quando a ação é benéfica para a melhoria da qualidade ambiental. \\
\hline Valor & Negativo: & Quando a ação resulta em dano à qualidade de um fator ambiental. \\
\hline \multirow[t]{2}{*}{ Dinâmica } & $\begin{array}{l}\text { Temporário: } \\
\text { Permanente: }\end{array}$ & $\begin{array}{l}\text { Quando permanece por um determinado tempo, após a realização da } \\
\text { ação. } \\
\text { Quando a ação é executada e os efeitos não cessamde se manifestar } \\
\text { em um determinado horizonte conhecido. }\end{array}$ \\
\hline & $\begin{array}{l}\text { Cíclico: } \\
\text { Imediato: }\end{array}$ & $\begin{array}{l}\text { O efeito se faz sentir em determinados períodos. } \\
\text { Surge no momento em que a ação está sendo realizada. }\end{array}$ \\
\hline \multirow[b]{2}{*}{ Tempo } & Curto prazo & Surge em curto prazo \\
\hline & $\begin{array}{l}\text { Médio prazo: } \\
\text { Longo prazo: }\end{array}$ & $\begin{array}{l}\text { Quando o efeito se manifesta após certo tempo quea ação foi } \\
\text { realizada. } \\
\begin{array}{c}\text { Quando o efeito se manifesta um longo tempo depois de } \\
\text { decorrido um certo tempo após a ação. }\end{array}\end{array}$ \\
\hline \multirow[t]{2}{*}{ Espaço } & Regional: & $\begin{array}{l}\text { Quando o efeito se manifesta um longo tempo depois de decorrido um } \\
\text { certo tempo após a ação. } \\
\text { Quando a ação afeta apenas o próprio local e suas imediações. }\end{array}$ \\
\hline & Nacional: & Quando a ação afeta apenas o próprio local e suas imediações. \\
\hline
\end{tabular}

Legenda: D: Direto; I: Indireto; R: Reversível; I: Irreversível; P: Positivo; N: Negativo; T: Temporário; P: Permanente; C: Cíclico; IM: Imediato; CP: Curto Prazo; MP: Médio Prazo; LP: Longo Prazo; L: Local; RE: Regional; NA: Nacional. Fonte: Sanchez (2008)

\section{Resultados e Discussão}

Com o estudo realizado identificou-se impactos dos meios abiótico, biótico e antrópico. Com isso foram listados através da metodologia "Checklist" os seguintes impactos: desmatamento, perda de biodiversidade, proliferação de vetores, erosão, desvalorização imobiliária, desconforto ambiental, poluição visual, voçoroca, contaminação do solo e poluição do ar, invasão de plantas exóticas e queimadas. 
Tabela 2. Avaliação qualitativa dos impactos dos Meios Bióticos e Meios Antrópicos.

\section{IMPACTOS}

IMPACTOS MEIO BIÓTICO MEIO ANTRÓPICO

\begin{tabular}{|c|c|c|c|c|}
\hline & Flora & Fauna & Saúde Pública & Prob. Econ. \\
\hline Desmatamento & $\begin{array}{l}\mathrm{D}, \mathrm{R}, \mathrm{N}, \mathrm{PR}, \\
\quad \mathrm{IM}, \mathrm{RE}\end{array}$ & $\begin{array}{l}\mathrm{D}, \mathrm{R}, \mathrm{N}, \mathrm{PR}, \\
\quad \mathrm{IM}, \mathrm{RE}\end{array}$ & $\begin{array}{c}\mathrm{I}, \mathrm{R}, \mathrm{N}, \mathrm{T}, \mathrm{CP} \\
\text { LO }\end{array}$ & $\mathrm{I}, \mathrm{R}, \mathrm{N}, \mathrm{T}, \mathrm{CP}, \mathrm{LO}$ \\
\hline $\begin{array}{c}\text { Perda de } \\
\text { biodiversidade }\end{array}$ & $\begin{array}{c}\mathrm{D}, \mathrm{R}, \mathrm{N}, \mathrm{PR}, \\
\mathrm{IM}, \mathrm{RE}\end{array}$ & $\begin{array}{c}\text { D, R, N, PR, } \\
\text { IM, RE }\end{array}$ & $\begin{array}{c}\mathrm{D}, \mathrm{R}, \mathrm{N}, \mathrm{T}, \\
\mathrm{CP}, \mathrm{LO}\end{array}$ & $\mathrm{D}, \mathrm{R}, \mathrm{N}, \mathrm{T}, \mathrm{CP}, \mathrm{LO}$ \\
\hline $\begin{array}{l}\text { Proliferaçãode } \\
\text { vetores }\end{array}$ & $\begin{array}{c}\text { I, R, N, PR, } \\
\text { IM, LO }\end{array}$ & $\begin{array}{l}\text { I, R, N, PR, } \\
\text { IM, LO }\end{array}$ & $\begin{array}{c}\text { I, R, N, T, IM, } \\
\text { LO }\end{array}$ & $\mathrm{I}, \mathrm{R}, \mathrm{N}, \mathrm{T}, \mathrm{CP}, \mathrm{LO}$ \\
\hline $\begin{array}{l}\text { Transmissãode } \\
\text { doenças }\end{array}$ & $\begin{array}{c}\text { I, R, N, PR, } \\
\text { CP, LO }\end{array}$ & $\begin{array}{c}\mathrm{I}, \mathrm{R}, \mathrm{N}, \mathrm{PR}, \\
\mathrm{CP}, \mathrm{LO}\end{array}$ & $\begin{array}{c}\text { I, R, N, PR, } \\
\text { IM, LO }\end{array}$ & $\mathrm{I}, \mathrm{R}, \mathrm{N}, \mathrm{T}, \mathrm{CP}, \mathrm{LO}$ \\
\hline Erosão & $\begin{array}{c}\text { I, R, N, PR, } \\
\text { IM, RE }\end{array}$ & $\begin{array}{c}\mathrm{I}, \mathrm{R}, \mathrm{N}, \mathrm{PR}, \\
\quad \mathrm{CP}, \mathrm{RE}\end{array}$ & $\begin{array}{c}\text { I, R, N, T, IM, } \\
\text { LO }\end{array}$ & $\mathrm{I}, \mathrm{R}, \mathrm{N}, \mathrm{T}, \mathrm{CP}, \mathrm{LO}$ \\
\hline $\begin{array}{c}\text { Desvalorização } \\
\text { imobiliária }\end{array}$ & $\begin{array}{c}\mathrm{I}, \mathrm{R}, \mathrm{N}, \mathrm{T}, \mathrm{CP} \\
\text { LO }\end{array}$ & $\begin{array}{c}\mathrm{I}, \mathrm{R}, \mathrm{N}, \mathrm{T}, \mathrm{CP} \\
\text { LO }\end{array}$ & $\begin{array}{c}\mathrm{D}, \mathrm{R}, \mathrm{N}, \mathrm{T}, \\
\mathrm{CP}, \mathrm{LO}\end{array}$ & $\mathrm{D}, \mathrm{R}, \mathrm{N}, \mathrm{T}, \mathrm{CP}, \mathrm{LO}$ \\
\hline $\begin{array}{c}\text { Desconforto } \\
\text { ambiental }\end{array}$ & $\begin{array}{l}\text { I, R, N, PR, } \\
\text { CP, RE }\end{array}$ & $\begin{array}{c}\mathrm{D}, \mathrm{R}, \mathrm{N}, \mathrm{PR}, \\
\quad \mathrm{CP}, \mathrm{RE}\end{array}$ & $\begin{array}{c}\mathrm{D}, \mathrm{R}, \mathrm{N}, \mathrm{T}, \\
\mathrm{CP}, \mathrm{LO}\end{array}$ & $\mathrm{D}, \mathrm{R}, \mathrm{N}, \mathrm{T}, \mathrm{CP}, \mathrm{LO}$ \\
\hline Poluição visual & $\begin{array}{c}\text { I, R, N, T, IM, } \\
\text { LO }\end{array}$ & $\begin{array}{c}\text { I, R, N, T, IM, } \\
\text { LO }\end{array}$ & $\begin{array}{c}\mathrm{D}, \mathrm{R}, \mathrm{N}, \mathrm{T}, \\
\mathrm{IM}, \mathrm{LO}\end{array}$ & $\mathrm{D}, \mathrm{R}, \mathrm{N}, \mathrm{T}, \mathrm{IM}, \mathrm{LO}$ \\
\hline Voçoroca & $\begin{array}{c}\mathrm{D}, \mathrm{R}, \mathrm{N}, \mathrm{PR}, \mathrm{IM}, \mathrm{L} \\
\mathrm{O}\end{array}$ & $\begin{array}{c}\mathrm{D}, \mathrm{IR}, \mathrm{N}, \mathrm{PR}, \mathrm{IM}, \mathrm{L} \\
\mathrm{O}\end{array}$ & $\begin{array}{c}\text { I, R, N, T, } \\
\text { CP,LO }\end{array}$ & $\mathrm{I}, \mathrm{R}, \mathrm{N}, \mathrm{T}, \mathrm{CP}, \mathrm{LO}$ \\
\hline $\begin{array}{c}\text { Contaminação do } \\
\text { solo }\end{array}$ & $\begin{array}{c}\mathrm{D}, \mathrm{R}, \mathrm{N}, \mathrm{PR}, \mathrm{IM}, \\
\text { LO }\end{array}$ & $\begin{array}{c}\mathrm{I}, \mathrm{R}, \mathrm{N}, \mathrm{PR}, \mathrm{CP}, \\
\text { LO }\end{array}$ & $\begin{array}{c}\text { I, R, N, PR, } \\
\text { CP, RE }\end{array}$ & $\mathrm{I}, \mathrm{T}, \mathrm{N}, \mathrm{T}, \mathrm{CP}, \mathrm{LO}$ \\
\hline Poluição do ar & $\begin{array}{c}\mathrm{I}, \mathrm{R}, \mathrm{N}, \mathrm{PR}, \mathrm{CP} \\
\mathrm{RE}\end{array}$ & $\begin{array}{c}\mathrm{I}, \mathrm{R}, \mathrm{N}, \mathrm{PR}, \mathrm{CP} \\
\mathrm{LO}\end{array}$ & $\begin{array}{c}\text { I, R, N, T, } \\
\text { CP, LO }\end{array}$ & I, R, N, T, CP, LO \\
\hline
\end{tabular}

Legenda: D: Direto; I: Indireto; R: Reversível; I: Irreversível; P: Positivo; N: Negativo; T: Temporário; P: Permanente; C: Cíclico; IM: Imediato; CP: Curto Prazo; MP: Médio Prazo; LP: Longo Prazo; L: Local; RE: Regional; NA: Nacional. Fonte: Autores. 
Tabela 3. Avaliação qualitativa dos impactos dos Meio Físico.

IMPACTOS

\begin{tabular}{|c|c|c|c|}
\hline IMPACTOS & MEIO ABIÓTICO & & \\
\hline & Solo & $\begin{array}{l}\text { Recursos } \\
\text { hídricos }\end{array}$ & Ar \\
\hline Desmatamento & $\begin{array}{c}\mathrm{D}, \mathrm{R}, \mathrm{N}, \mathrm{PR}, \\
\mathrm{IM}, \mathrm{LO}\end{array}$ & $\mathrm{I}, \mathrm{R}, \mathrm{N}, \mathrm{T}, \mathrm{CP}, \mathrm{LO}$ & $\mathrm{I}, \mathrm{R}, \mathrm{N}, \mathrm{T}, \mathrm{CP}, \mathrm{LO}$ \\
\hline Queimadas & $\mathrm{D}, \mathrm{R}, \mathrm{N}, \mathrm{T}, \mathrm{CP}, \mathrm{RE}$ & $\mathrm{D}, \mathrm{R}, \mathrm{N}, \mathrm{T}, \mathrm{CP}, \mathrm{LO}$ & $\mathrm{D}, \mathrm{R}, \mathrm{N}, \mathrm{T}, \mathrm{CP}, \mathrm{RE}$ \\
\hline $\begin{array}{c}\text { Perda de } \\
\text { biodiversidade }\end{array}$ & $\begin{array}{c}\mathrm{D}, \mathrm{R}, \mathrm{N}, \mathrm{PR}, \\
\mathrm{CP}, \mathrm{LO}\end{array}$ & $\mathrm{D}, \mathrm{R}, \mathrm{N}, \mathrm{T}, \mathrm{CP}, \mathrm{LO}$ & $\mathrm{D}, \mathrm{R}, \mathrm{N}, \mathrm{T}, \mathrm{CP}, \mathrm{LO}$ \\
\hline $\begin{array}{l}\text { Proliferaçãode } \\
\text { vetores }\end{array}$ & $\mathrm{I}, \mathrm{R}, \mathrm{N}, \mathrm{PR}, \mathrm{CP}, \mathrm{LO}$ & $\mathrm{I}, \mathrm{R}, \mathrm{N}, \mathrm{T}, \mathrm{CP}, \mathrm{LO}$ & $\mathrm{I}, \mathrm{R}, \mathrm{N}, \mathrm{T}, \mathrm{CP}, \mathrm{LO}$ \\
\hline $\begin{array}{l}\text { Transmissãode } \\
\text { doenças }\end{array}$ & $\mathrm{I}, \mathrm{R}, \mathrm{N}, \mathrm{PR}, \mathrm{CP}, \mathrm{LO}$ & $\mathrm{I}, \mathrm{R}, \mathrm{N}, \mathrm{PR}, \mathrm{IM}, \mathrm{LO}$ & $\mathrm{I}, \mathrm{R}, \mathrm{N}, \mathrm{T}, \mathrm{CP}, \mathrm{LO}$ \\
\hline Erosão & $\mathrm{I}, \mathrm{R}, \mathrm{N}, \mathrm{PR}, \mathrm{IM}, \mathrm{LO}$ & $\mathrm{I}, \mathrm{R}, \mathrm{N}, \mathrm{PR}, \mathrm{CP}, \mathrm{LO}$ & $\mathrm{I}, \mathrm{R}, \mathrm{N}, \mathrm{PR}, \mathrm{CP}, \mathrm{LO}$ \\
\hline $\begin{array}{c}\text { Desvalorização } \\
\text { imobiliária }\end{array}$ & $\mathrm{I}, \mathrm{R}, \mathrm{N}, \mathrm{T}, \mathrm{CP}, \mathrm{LO}$ & $\mathrm{I}, \mathrm{R}, \mathrm{N}, \mathrm{T}, \mathrm{CP}, \mathrm{LO}$ & $\mathrm{I}, \mathrm{R}, \mathrm{N}, \mathrm{T}, \mathrm{CP}, \mathrm{LO}$ \\
\hline $\begin{array}{l}\text { Invasão de } \\
\text { plantas } \\
\text { exóticas }\end{array}$ & $\mathrm{I}, \mathrm{R}, \mathrm{N}, \mathrm{PR}, \mathrm{CP}, \mathrm{RE}$ & $\mathrm{I}, \mathrm{R}, \mathrm{N}, \mathrm{PR}, \mathrm{CP}, \mathrm{LO}$ & $\mathrm{I}, \mathrm{R}, \mathrm{N}, \mathrm{PR}, \mathrm{CP}, \mathrm{LO}$ \\
\hline Voçoroca & $\begin{array}{c}\mathrm{D}, \mathrm{R}, \mathrm{N}, \mathrm{PR}, \\
\mathrm{IM}, \mathrm{LO}\end{array}$ & I, R, N, T, CP,LO & $\mathrm{I}, \mathrm{R}, \mathrm{N}, \mathrm{T}, \mathrm{CP}, \mathrm{LO}$ \\
\hline $\begin{array}{l}\text { Contaminaçãodo } \\
\text { solo }\end{array}$ & $\begin{array}{c}\mathrm{D}, \mathrm{R}, \mathrm{N}, \mathrm{PR}, \\
\mathrm{IM}, \mathrm{LO}\end{array}$ & $\mathrm{I}, \mathrm{R}, \mathrm{N}, \mathrm{PR}, \mathrm{CP}, \mathrm{RE}$ & $\mathrm{I}, \mathrm{T}, \mathrm{N}, \mathrm{T}, \mathrm{CP}, \mathrm{LO}$ \\
\hline Poluição do ar & $\mathrm{I}, \mathrm{R}, \mathrm{N}, \mathrm{PR}, \mathrm{CP}, \mathrm{RE}$ & $\mathrm{I}, \mathrm{R}, \mathrm{N}, \mathrm{T}, \mathrm{CP}, \mathrm{LO}$ & $\mathrm{I}, \mathrm{R}, \mathrm{N}, \mathrm{T}, \mathrm{CP}, \mathrm{LO}$ \\
\hline
\end{tabular}

Legenda: D: Direto; I: Indireto; R: Reversível; I: Irreversível; P: Positivo; N: Negativo; T: Temporário; P: Permanente; C: Cíclico; IM: Imediato; CP: Curto Prazo; MP: Médio Prazo; LP: Longo Prazo; L: Local; RE: Regional; NA: Nacional. Fonte: Autores.

Na Tabela 3 é possível observar que a maior parte dos impactos registrados são de parâmetros indiretos resultantes do desmatamento e descarte incorreto de resíduos sólidos na margem da rodovia.

Todos os impactos registrados foram considerados negativos (Tabelas 2 e 3), pois estes acabam por resultar em dano à qualidade dos meios abiótico, biótico e antrópico.

Foi identificado na área de estudo uma desvalorização imobiliária (Tabela: 1), (Figura: 2) como consequência da presença de voçorocas, erosão e deposição inadequada de resíduos sólidos que acabam por desencadear uma série de problemas 
ambientais, como por exemplo, a destruição de terras cultiváveis e/ou cultivadas, equipamentos urbanos e obras civis, além disso esse fenômeno pode conduzir boa parte do material removido aos cursos d'água, propiciando condições extremamente favoráveis a enchentes (Sá, 2001; Rocha \& Nishiyama, 2012).

Figura 2. Resíduos sólidos e Voçorocas.

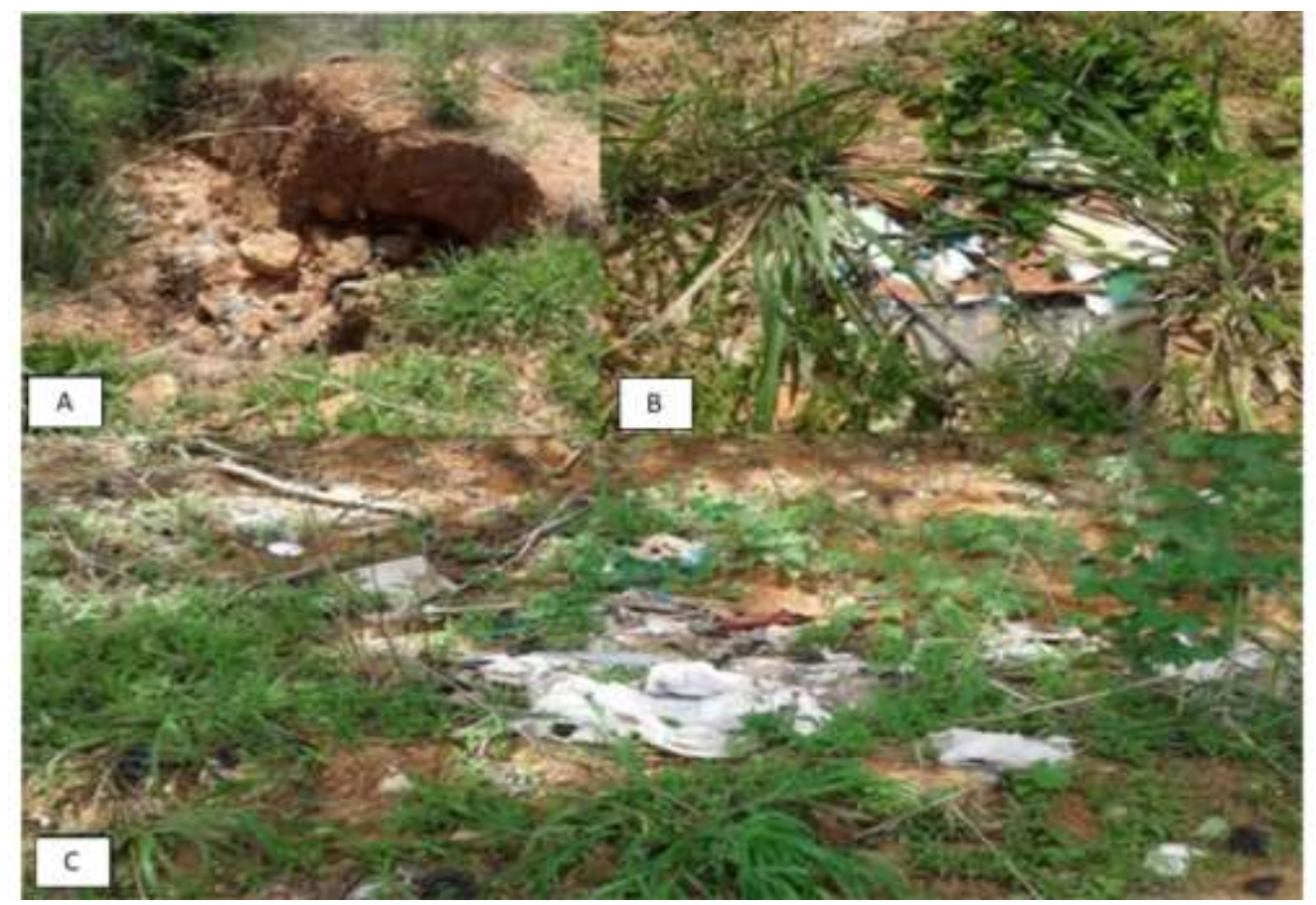

Legenda: (A) formação de voçorocas, (B) Descarte irregula de entulho e (C) Resíduos sólidos. Fonte: Autores.

Para Roberto (2013) um fator que contribui diretamente para a presença das voçorocas está associado a paisagens de onde foi retirada a sua cobertura vegetal. Nas imagens expostas acima, a água de escoamento superficial ao percolar linearmente no solo, atinge o lençol freático e compromete a estabilidade da área e gera a formação de voçorocas. Além disso, outros fatores são condicionantes ao surgimento desse fenômeno, dentre os quais podem ser destacados como agentes antrópicos as queimadas, o desmatamento e o manejo inadequado de plantações, elementos geológicos passivos e ativos, fatores pedológicos, climáticos ativos e passivos e geomorfológicos (Bacellar, 2006). O uso inadequado do solo atua na fragilidade e a no potencial impacto que o solo pode vir a sofrer (Braga et ai, 2021). Nestes casos, as áreas estão ocupadas inadequadamente, o que demanda sua readequação.

A presença de vetores foi registrada em água parada (Figura: 3) que se encontrava em embalagens descartadas à margem da rodovia e em pequenas poças d'água. Estes agentes, por sua vez, encontram nestes ambientes um lugar propício para se reproduzir e, consequentemente, transmitir doenças ao ser humano. Quando o lixo é disposto de forma inadequada, os problemas ambientais são inevitáveis, em um estudo demonstrado por Ribeiro (2010) dentre todas as doenças destacadas a de maior ocorrência foi a dengue. 
Figura 3. Resíduos sólidos encontrados com água parada e poças de água.

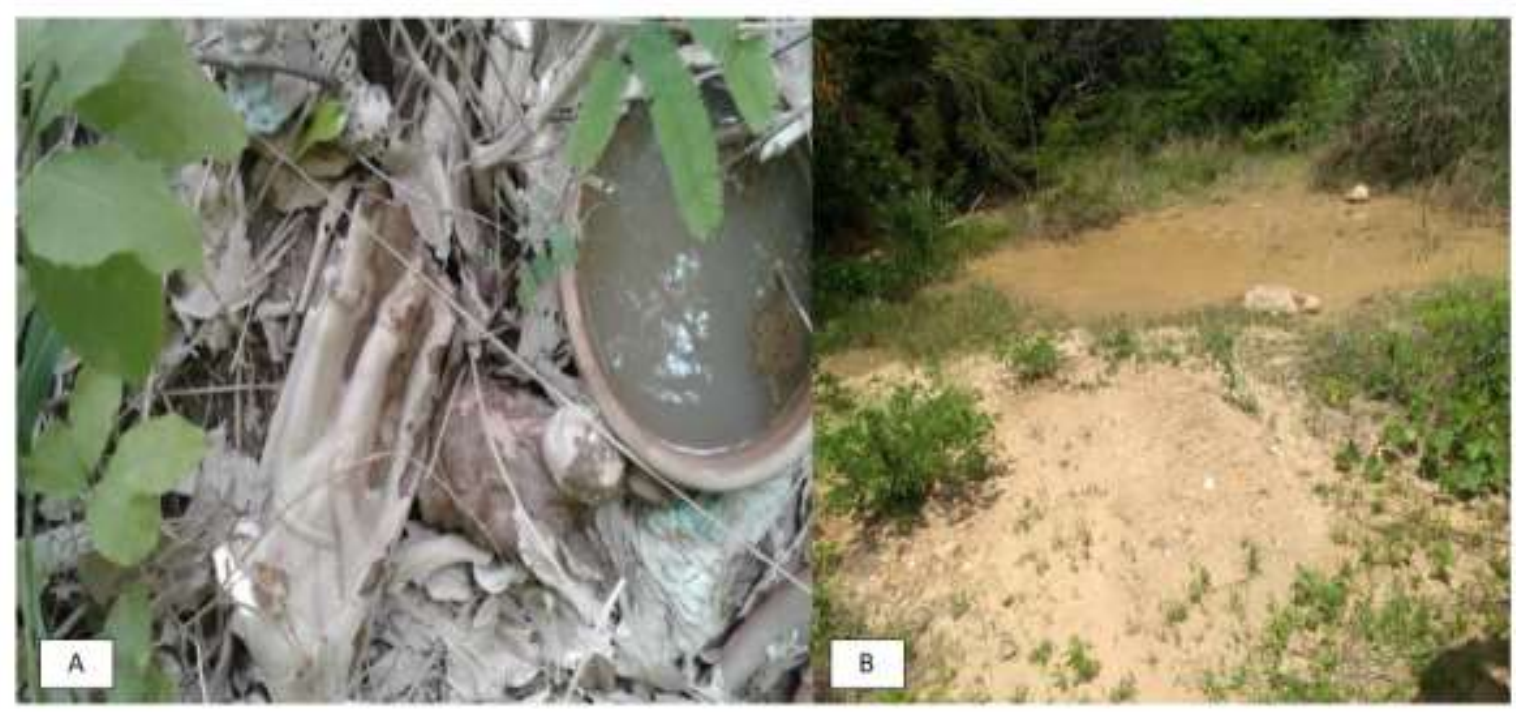

Legenda: (A) embalagens pláticas com acúmulo de água e (B) Poçcas de água propicias para a ploriferação de vetores. Fonte: Autores.

Além disso, um impacto registrado e considerado perigoso ao meio biótico e meio antrópico foi a contaminação do solo resultante ainda da presença de resíduos sólidos depositados de forma inadequada nesses ambientes, gerando também outros impactos, como poluição visual e poluição do ar (figura 06). Foi identificado na área de estudo uma desvalorização imobiliária como consequência da presença de voçorocas, erosão e deposição. A deposição de resíduos sólidos descartados de forma inadequada pode criar condições propícias para a geração de chorume que se torna um líquido altamente poluidor que pode trazer danos ao meio ambiente, como a contaminação do solo, do ar e das águas superficiais e subterrâneas, além da proliferação de vetores de doenças influenciando negativamente a qualidade ambiental e a saúde da população (Pereira \& Curi, 2013).

Observou-se no local algumas áreas sem cobertura vegetal em decorrência de porções de lixos espalhados. Além do mais, verificou-se a presença de algumas espécies invasoras, como a mangueira (Mangifera indica), que de alguma forma acaba competindo com as espécies nativas. Atualmente, as plantas exóticas invasoras são consideradas a segunda maior ameaça mundial da biodiversidade, modificando as características naturais do ecossistema ( de Carvalho França et al, 2020).

Verificou-se que a maior parte da área foi desmatada para construção da rodovia nova e para expansão imobiliária. A fase de operação de uma rodovia gera vários impactos e modificações no ambiente original, como poluição do ar, através da emissão de gases na atmosfera, e com o consequente fluxo de veículos na rodovia, poluição da água, do solo e erosão (Gallardo, 2004).

Entre os impactos físicos negativos, constatou-se em algumas margens da rodovia, áreas queimadas, uma prática primitiva da agricultura que tem por finalidade facilitar a limpeza do terreno para plantação. As queimadas causam prejuízos irreparáveis para a biodiversidade, ao ciclo hidrológico e ao ciclo de carbono na atmosfera, além de ameaçar espécies de extinção e causar a erosão e empobrecimento do solo, é uma das principais causas de incêndios florestais (Cabral, et al, 2013).

\section{Considerações Finais}

A degradação ambiental da área estudada causa inúmeros problemas ao ambiente: impermeabilização do solo que causa as voçorocas, o aumento da ocupação urbana que acarreta inúmeros impactos visuais, químicos e principalmente físicos a esses ambientes. Como resultados indesejáveis observou-se um grande número de resíduos sólidos que contaminam os lençóes freáticos, bem como servem de repositórios de larvas de insetos de importância epidemiológica.

Partindo do diagnóstico desse trabalho, ressalta-se a importância de políticas públicas que proponham medidas de 
restauração e implementação de zonas de transição, para que esses ambientes possam ser recuperados após um grande estresse ambiental como o foi pela construção da rodovia. Neste sentido, a deterioração ambiental uma vez restaurada, pode trazer de volta uma melhor qualidade de vida para a comunidade com um todo, pois na ausência de intervenções o prognóstico é de contínua deterioração de suas propriedades bióticas e abióticas.

Fa-se necessario a realização de atividades de monitoramento ambiental dos órgãos públicos responsáveis, bem como a organização de ações de educação ambiental, para que em um futuro p'roximo esse mesmo ambiente esteja livre da poluição e degradação ambiental.

\section{Referências}

Bacellar, L. (2006). de AP Processos de formação de voçorocas e medidas preventivas e corretivas. UFOP.

Braga, S. E., Caldas, A. M., Rizzi Neto, E., Silva, L. J. de S., Perônico, A. M. B. L., Wanderley, R. A., Freitas, P. M. L. de, Lima, J. R. de, Cunha, C. R. R. O. da, \& Abreu, B. S. (2021). Conflito ambiental de uso do solo na bacia hidrográfica de Tapacurá-PE. Pesquisa, Sociedade e Desenvolvimento , 10 (2), e52310212833. https://doi.org/10.33448/rsd-v10i2.12833

Brasil. (1997). Decreto de 04 de agosto de 1997. Dispõe sobre a criação da Área de Proteção Ambiental da chapada do Araripe, nos Estados do Ceará, Pernambuco e Piauí e dá outras providências. Diário Oficial. Brasília.

Brito, R. S. C., \& Valverde, T. S. (2020). O Projeto de Lei n $6.299 / 2002$ e as violações socioambientais.

Cabral, A. L. A., Moras Filho, L. O., \& Borges, L. A. C. (2013). Uso Do Fogo Na Agricultura: Legislação, Impactos Ambientais E Realidade Na Amazônia. Periódico Eletrônico Fórum Ambiental da Alta Paulista, 9(5).

Correia, D. B., Freita, F. R. V., de Oliveira, A. H., Correia, D. B., Teixeira, P. H. R., Soares, T. R. C., ... \& Pinheiro, A. P. (2020). Etnobiologia do caranguejo Kingsleya attenboroughi Pinheiro \& Santana 2016 na Área de Proteção Ambiental Chapada do Araripe. Research, Society and Development, 9(12), e19091210827-e19091210827.

de Carvalho França1 ${ }^{1}$, A., Silva, R. G., Matricardi¹, E. A. T., Sampaio, A. B., \& Guimarães, T. C. S. (2020). Susceptibilidade à invasão das Unidades de Conservação federais por espécies exóticas invasoras da flora terrestre. Agropecuária Científica No Semiárido, 16(3), 126-133.

Gallardo, A. L. C. F. (2004). Análise das práticas de gestão ambiental da construção da pista descendente da Rodovia dos Imigrantes (Doctoral dissertation, Universidade de São Paulo).

Instituto Brasileiro de Geografia e Estatística (IBGE) 2020. https://www.ibge.gov.br/cidades-e-estados/ce/crato.html

Leff, E., \& Vieira, P. F. (2001). Epistemologia ambiental (Vol. 2). Cortez.

Pereira, S. S., \& Curi, R. C. (2013). Modelos de gestão integrada dos resíduos sólidos urbanos: a importância dos catadores de materiais recicláveis no processo de gestão ambiental. Gestão sustentável dos recursos naturais: uma abordagem participativa [online]. Campina Grande: EDUEPB, $149-172$.

Pinheiro, G. M., \& Braga Branquinho, F. T., Felzenszwalb, I. (2011). Uma análise contextual do funcionamento efetivo e participação popular em uma unidade de conservação: o caso da área de proteção ambiental de Petrópolis (Rio de Janeiro: Brasil). Sociedade \& Natureza, 23(2), 323-334.

Ponce, V. (2011). La Matriz de Leopold para la evaluación del impacto ambiental. http://ponce. sdsu. edu/la_matriz_de_leopold. html.

Reis, D., Friede, R., \& Lopes, F. H. P. (2018). Política nacional de resíduos sólidos (Lei no 12.305/2010) e educação ambiental. Revista Interdisciplinar de Direito, 14(1), 99-111.

Ribeiro, J. W., \& Rooke, J. M. S. (2010). Saneamento básico e sua relação com o meio ambiente e a saúde pública. Monografia de Especialização em Análise Ambiental, Universidade Federal de Juiz de Fora, Minas Gerais, Brasil. 36p, 2010.

Roberto, D. C. (2013). Identificação de Fatores que contribuíram para a Formação de uma Voçoroca existente na Fazenda Experimental Agro-Ecológica Izabela Hendrix, Sabará/MG. Acervo da Iniciação Científica, (1).

Rocha, L., \& Nishiyama, L. (2012). Qualidade das águas superficiais e subterrâneas em voçoroca ocupada por resíduos sólidos urbanos-Uberlândia (MG). Observatorium: Revista Eletrônica de Geografia, 4(12).

Sá, C. (2001). Mapeamento de declividades voltado para o controle de processos erosivos em sítios florestais. Monografia apresentada à Universidade Federal de Minas Gerais. 35p. http://www.csr.ufmg.br/geoprocessamento/centrorecursos/3cursopub/alvessa2000.pdf

Sánchez, L. E. (2015). Avaliação de impacto ambiental. Oficina de textos.

Silva, J. L. C., Vidal, C. A. S., Barros, L. M., \& Freita, F. R. V. (2018). Aspectos da degradação ambiental no Nordeste do Brasil. Revista Gestão \& Sustentabilidade Ambiental, 7(2), 180-191.

Tagliapietra, O. M., \& Carniatto, I. (2019). A interdisciplinariedade na Educação Ambiental como instrumento para a consolidação do Desenvolvimento Sustentável. Revista Brasileira de Educação Ambiental (RevBEA), 14(3), 75-90.

Tristão, M. (2002). As dimensões e os desafios da educação ambiental na sociedade do conhecimento. Educação ambiental: abordagens múltiplas. Porto Alegre: Artmed, 169-173. 